University of Nebraska - Lincoln

DigitalCommons@University of Nebraska - Lincoln

\title{
Sex effects on life span and senescence in the wild when dates of birth and death are unknown
}

\author{
Felix Zajitschek \\ University of New South Wales \\ Chad Brassil \\ University of Nebraska - Lincoln, cbrassil@unl.edu \\ Russell Bonduriansky \\ University of New South Wales \\ Robert C. Brooks \\ University of New South Wales
}

Follow this and additional works at: https://digitalcommons.unl.edu/bioscifacpub

Part of the Biology Commons

Zajitschek, Felix; Brassil, Chad; Bonduriansky, Russell; and Brooks, Robert C., "Sex effects on life span and senescence in the wild when dates of birth and death are unknown" (2009). Faculty Publications in the Biological Sciences. 316.

https://digitalcommons.unl.edu/bioscifacpub/316

This Article is brought to you for free and open access by the Papers in the Biological Sciences at DigitalCommons@University of Nebraska - Lincoln. It has been accepted for inclusion in Faculty Publications in the Biological Sciences by an authorized administrator of DigitalCommons@University of Nebraska - Lincoln. 


\title{
Sex effects on life span and senescence in the wild when dates of birth and death are unknown
}

\author{
Felix Zajitschek, ${ }^{1,3}$ Chad E. Brassil, ${ }^{2}$ Russell Bonduriansky, ${ }^{1}$ and Robert C. Brooks ${ }^{1}$ \\ ${ }^{1}$ Evolution and Ecology Research Centre and School of Biological, Earth and Environmental Sciences, \\ University of New South Wales, Kensington, Sydney, NSW 2052 Australia \\ ${ }^{2}$ School of Biological Sciences, University of Nebraska, Lincoln, Nebraska 68588-0118 USA
}

\begin{abstract}
Males and females allocate and schedule reproductive effort in very different ways. Because the timing and amount of reproductive effort influence survival and thus the optimization of life histories, mortality and senescence are predicted to be sex specific. However, age-specific mortality rates of wild animals are often difficult to quantify in natural populations. Studies that report mortality rates from natural populations are, therefore, almost entirely confined to long-lived, easy-to-track species such as large mammals and birds. Here, we employ a novel approach using capture-mark-recapture data from a wild population of black field crickets (Teleogryllus commodus) to test for sex differences in demographic aging. In this species, the age of captured adults cannot be readily determined, and animals cannot be reliably captured or observed every night, resulting in demographic data on individuals whose dates of birth and death are unknown. We implement a recently developed life-table analysis for wild-caught individuals of unknown age, in combination with a well-established capture-mark-recapture methodology that models probabilistic dates of death. This unified analytical framework makes it possible to test for aging in wild, hard-totrack animals. Using these methods to fit Gompertz models of age-specific mortality, we show that male crickets have higher mortality rates throughout life than female crickets. Furthermore, males and females both exhibit increasing mortality rates with age, indicating senescence, but the rate of senescence is not sex specific. Thus, observed sex differences in longevity are probably due to differences in baseline mortality rather than aging. Our findings illustrate the complexity of the relationships between sex, background mortality, and senescence rate in wild populations, showing that the elevated mortality rate of males need not be coupled with an elevated rate of aging.
\end{abstract}

Key words: aging; black field cricket; capture-mark-recapture; Gompertz; natural population; open population; sex differences; sexual dimorphism; Teleogryllus commodus.

\section{INTRODUCTION}

Longevity and mortality patterns differ between males and females in many species due to sex differences in background mortality and time of onset and the rate of aging (Promislow and Harvey 1990, Promislow 1991, Fox et al. 2003, Magwere et al. 2004, Liker and Szekely 2005, Kruger and Nesse 2006, Clutton-Brock and Isvaran 2007). Such sex differences are commonly thought to be due to sex differences in the timing and costs of reproductive effort (Williams 1957, Kirkwood and Rose 1991, Clutton-Brock and Isvaran 2007). In addition, sexual conflict (Parker 1979, Arnqvist and Rowe 2005) has been suggested as a possible explanation of sex-specific life span and mortality patterns and even as an agent for the evolution of senescence itself (Svensson and Sheldon 1998, Promislow 2003).

Manuscript received 8 January 2008; revised 5 September 2008; accepted 12 September 2008. Corresponding Editor: J. M. Fryxell.

${ }^{3}$ Present address: Station d'Ecologie Expérimentale du CNRS Moulis (USR 2936), 09200 St.-Girons, France.

E-mail: felix.zajitschek@EcoEx-Moulis.cnrs.fr
Observed mortality rates can be partitioned into different sources: "extrinsic" mortality hazards such as predation originate with an external agent, whereas "intrinsic" causes of death come about due to the way in which age-specific trade-offs in resource allocation for various purposes within the body are resolved (Williams and Day 2003, Bronikowski and Promislow 2005). Extrinsic and intrinsic mortality influence one another over evolutionary time because age-specific allocation patterns are shaped by the levels of extrinsic mortality hazards and thus the age-specific strength of selection on the population, and because intrinsic factors such as condition can alter the vulnerability of an individual to extrinsic mortality risks (Williams 1957, Hamilton 1966, Charlesworth 1994, Partridge and Barton 1996, Carnes and Olshansky 1997). Therefore, in order to understand sex differences in longevity and aging, it is necessary to tease apart the interrelated issues of sex-dependent extrinsic mortality in the environment where adaptation has taken place, sex differences in the nature and timing of reproductive effort, and the sources of sex-dependent selection. 
Recently, considerable progress has been made toward understanding the effects of sexual selection on life span and aging (Carranza et al. 2004, Carranza and Pérez-Barbería 2007, Clutton-Brock and Isvaran 2007). In species in which sexual selection has driven the elaboration of male ornaments, displays, or weapons, males are expected to experience greater overall mortality than females due to high predation or parasitism, elevated levels of damage from fights, and the energetic costs of expressing these costly traits (Endler 1980, Crespi 1988, Owen-Smith 1993, Zuk and Kolluru 1998). Under these circumstances, the classic evolutionary theory of aging predicts an earlier onset of aging and a more rapid aging in males (Medawar 1952, Williams 1957; but see Abrams and Ludwig 1995, Williams et al. 2006). On the contrary, males might show an aging rate that is much more similar to that of females when agedependent fitness components such as calling effort peak at an intermediate age or even increase throughout life (Graves 2007). This would lead to increased selection against somatic deterioration until the peak age is reached.

Research programs that seek to resolve the adaptive basis of mortality and aging patterns need to include an understanding of what the patterns are and what forces are at play within the natural populations where adaptation took place, as a necessary context for laboratory studies where most external hazards and stresses are excluded (but see Partridge and Barton 1996, Ricklefs 2000, Williams et al. 2006). Relative to natural environments, laboratory conditions typically yield vastly reduced extrinsic mortality rates and increased life expectancy. Benign laboratory conditions also can have pronounced effects on the expression of aging, and such effects can be sex specific in nature (Kawasaki et al. 2008). This suggests that laboratory studies of aging and life span may yield misleading results. However, empiricists wishing to estimate age-dependent mortality rates and rates of senescence in natural populations are faced with several challenges: individual life spans for a cohort of animals that reflect the whole natural population usually have to be known in order to calculate a life table (Carey 2001). This might be achieved by monitoring animals from birth to death, but this is highly challenging in many species, with population size and range, life span, body size, cryptic lifestyle, and high dispersal rate all bearing on the problem. Consequently, the literature on demographic aging in the wild is strongly biased toward vertebrates (McDonald et al. 1996, Mysterud et al. 2001, Reznick et al. 2001, Orell and Belda 2002, Reid et al. 2003, Nussey et al. 2006, Toigo et al. 2007), due to the relative tractability of catching, marking, and monitoring individuals. By contrast, invertebrates are more amenable to laboratory studies, due to their short life span and easy and economic culture requirements (Finch 1994). Few studies of aging in the wild have been conducted on invertebrates (but see Bonduriansky and Brassil 2002,
Kawasaki et al. 2008) because of the difficulties of identifying individuals of known age and monitoring them until they die.

Capture-mark-recapture methodology allows the estimation of survival rates from records of marked and recaptured animals, where time of death of marked animals is normally not known (Pollock 1991). If the study population is "open" (i.e., births, deaths, and migration into and out of the populations can occur between sampling intervals), Cormack-Jolly-Seber models (CJS; Cormack 1964, Jolly 1965, Seber 1965) are very well suited to modeling survival probabilities (reviewed in Pollock 1991, Lebreton et al. 1992) and thus estimating life tables. To test for and characterize senescence in a population, estimated mortality rates have to be age specific (Charlesworth 1994). When it is not possible to determine the age of an animal at the time of entry into the study ("age at entry," i.e., at the time of first capture), age-specific mortality cannot be properly modeled in the CJS framework.

Crespin et al. (2006) used simulations to show that the time between first capture and last recapture could be used as a proxy for life span of marked Common Guillemots (Uria aalge), a cliff-dwelling species with high resighting and recapture rates. Although this approach is adequate when recapture probabilities are very high relative to life span, it is not likely to be the most suitable method when recapture rates are relatively low, as is often the case with capture-mark-recapture studies on animals that are cryptic or difficult to catch. A recently published analytical method addresses this problem more generally, allowing inferences about the true age-specific survival rates of a cohort of animals with unknown ages at entry, given their schedule of estimated time to death (Müller et al. 2004). To test for demographic aging, a mortality model like the Gompertz model (Gompertz 1825) can be implemented and its parameters can be estimated. However, the method of Müller et al. has the opposite limitation to the CJS method: it models uncertainty over birth dates but requires death dates to be known (as would be possible for a closely monitored population of large vertebrates). Here, we combine for the first time the CJS approach and the method developed by Müller et al. (2004), thus providing a powerful unified approach to the analysis of demographic aging and senescence in natural populations. We apply this method to test for sex-dependent mortality and aging in a field cricket, Teleogryllus commodus, which we have already established as a tractable model for studying how sex-dependent reproductive effort influences the evolution of life span and aging (Hunt et al. 2004, 2006, Zajitschek et al. 2007, Maklakov et al. 2008).

\section{Methods \\ Capture-recapture methods}

We collected 1554 adult black field crickets, Teleogryllus commodus, between 9 February 2006 and 4 
March 2006 from a pasture near Smith's Lake $\left(32^{\circ} 23^{\prime} \mathrm{S}\right.$, $\left.152^{\circ} 30^{\prime} \mathrm{E}\right)$, New South Wales, Australia. T. commodus is a univoltine cricket species, laying eggs that have the capacity to diapause (Browning 1954, Hogan 1966). For our capture-recapture study, crickets were collected at night, brought back to the lab, and marked the following day. For this, we used numbers laser-printed on white paper in 4-point Arial Narrow font, which we cut out with fine scissors, glued on to the dorsal side of the pronotum with clear nail polish (Maybelline, New York, New York, USA), and let dry for 30 minutes, during which we kept the marked cricket individually. Marking methods had been tested previously in the laboratory by keeping crickets marked using several different techniques for 30 days in a container that contained damp earth as substrate and stones and twigs to simulate potential environmental conditions that could affect adhesion. The paper-nail polish method described here yielded better results (none of the animals lost their marks) than other methods (e.g., honey bee labels, other adhesives) and thus satisfies the assumption of CJS models that marks are not lost during the experiment (Pollock 1991). After marking, crickets were released at the center of the sampling site on the night following capture (after that night's unmarked crickets had been captured).

The area in which we captured and recaptured the crickets measured $22 \times 40 \mathrm{~m}$ and was demarcated on two sides by a cow fence. Behind one fenced side was a $10 \mathrm{~m}$ wide road verge area covered with short-mown grass; behind the other fenced side was a cemetery, with equally short grass. The other two unfenced sides of the sampling area were not separated physically in any way from the rest of the field, which was in total several hundred meters long and wide. We only encountered the cows that were kept on this field twice in our sampling area during the whole experiment. The vegetation was a mixture of tall (up to $120 \mathrm{~cm}$ high) grasses, short grass patches, and some small sandy and bare-soil patches. We sampled marked crickets in the field every night between 11 February 2006 and 20 April 2006 (68 days), usually starting at 22:00 hours and continuing for an average of 88 person-minutes (=number of minutes of sampling per night $\times$ number of people that sampled). On each sampling occasion, we aimed to cover the whole of the sampling area, and adjusted the sampling area that an individual person had to cover according to the number of people available at a given night. We looked for crickets on the ground with the aid of strong headlamps and by carefully brushing grass aside and looking in potential hiding places near or under dense grass clusters.

Because we sampled an open population without any environmental barriers between the inside and outside of the sampling area, we checked for dispersal of marked crickets to the outside of the sampling area. We therefore searched for marked crickets within a perimeter of $10 \mathrm{~m}$ outside the sampling area on 23 nights for, on average, 31 person-minutes. We recaptured nine marked females within this $10-\mathrm{m}$ perimeter on eight of these nights, which corresponds to a recapture rate of $7.73 \times 10^{-6}$ females $(\text { person-minute) })^{-1} \cdot \mathrm{m}^{-2}$. In the main sampling area, the average recapture rate throughout the study was 22-fold higher: $1.69 \times 10^{-4}$ females.(person-minute) $)^{-1} \cdot \mathrm{m}^{-2}$. We found no males in the perimeter, while the rate of recapture in the main sampling area was $3.33 \times 10^{-5}$ males.(person-minute) $)^{-1} \cdot \mathrm{m}^{-2}$. On 15 out of 23 nights we did not recapture any marked crickets in the outside border.

In total 158 males and 496 females were recaptured at least once $(32.1 \%$ of males, $46.7 \%$ of females released in total) in the sampling area.

\section{Statistical methods}

The Gompertz model was used to estimate rates of senescence:

$$
m(x)=\alpha e^{\beta x}
$$

where the instantaneous per capita mortality rate $m$ is a function of the age $x$, with parameters for the background mortality rate $\alpha$ and the change in mortality rate with age $\beta$. Assuming organisms of known age, one could derive an expression for survival rates from Eq. 1 and estimate the senescence parameters in the Gompertz model. However, in this study we analyzed wildcaptured crickets of unknown age at first capture; therefore we based our analysis on the technique described by Müller et al. (2004). First, solving Eq. 1 yields the discrete-time expression for the fraction of individuals $l_{x}$ that remain alive at day $x$ :

$$
l_{x}=\frac{n_{x}}{n_{0}}=\exp \left[-\frac{\alpha}{\beta}\left(e^{\beta x}-1\right)\right]
$$

where $n_{x}$ is the number of crickets alive at age $x$. Assuming a stable age distribution, Müller et al. (2004) demonstrated that age distribution $c_{x}$ in a wild population will be identical to the distribution of deaths $d_{x}^{*}$ in a marked sample of individuals. This fundamental identity permits inferences about senescence patterns from data on time to death.

We incorporated this technique into classic markrecapture analysis that models a probabilistic death (Lebreton et al. 1992) as opposed to a known age at death. The mark-capture technique estimates $p$, the probability of observing an individual given that it is alive. Estimates of $p$ were scaled per person-minute of observation time to account for differences in capture effort on different days. The probability of observing a cricket on a given day with person-minute observations $\mu$ is

$$
1-(1-p)^{\mu}
$$

and temperature dependence was included via the inverse logit function: 


$$
1-\frac{1}{1+\exp [-(p+\tau T)]}
$$

where the temperature $T$ is included with the coefficient $\tau$. Essentially, we estimate senescence rates from individuals with an unknown date of birth and an unknown date of death by assuming a stable age distribution upon capture and an increasingly small chance of individuals remaining alive if we fail to recapture them on repeated days.

Following the convention of Müller et al. (2004), an asterisk is used to indicate parameters of the marked population of unknown age, and non-asterisk parameters indicate the true parameters of the wild population. The distribution of individuals in the marked wild population that die on each day, from day $x$ to the next discrete day, is $d_{x}^{*}$. The fraction of individuals that survive in the marked population from one day to the next, $s_{x}^{*}$, by definition, is the ratio of the fraction of individuals that survive from the day of marking to day $x+1$ over the fraction that survive to day $x$ :

$$
s_{x}^{*}=\frac{l_{x+1}^{*}}{l_{x}^{*}} .
$$

Here, $l_{x}^{*}$, the fraction of individuals that survive from the day of marking to day $x$, is a function of the distribution of deaths $d_{x}^{*}$ :

$$
l_{x}^{*}=1-\sum_{j=0}^{x-1} d_{j}^{*} .
$$

Using this relationship in Eq. 6, Eq. 5 can be framed in terms of $d_{x}^{*}$ :

$$
s_{x}^{*}=\frac{1-\sum_{j=0}^{x} d_{j}^{*}}{1-\sum_{j=0}^{x-1} d_{j}^{*}} .
$$

The key insight from the life-table methodology is that the distribution in the marked population of deaths on day $x$ since capture is equal to the stable age distribution in the wild population, which is equal to $l_{x}$ normalized by the sum of all $l_{x}$ values (Müller et al. 2004):

$$
d_{x}^{*}=\frac{l_{x}}{\sum_{j=0}^{\infty} l_{j}}
$$

Substituting Eq. 8 into Eq. 7 and rearranging yields an expression for the fraction of individuals that survive in the marked population as a function of senescence parameters from Eq. 2 in the wild population:

$$
s_{x}^{*}=\frac{\sum_{j=0}^{\infty} l_{j}-\sum_{j=0}^{x} l_{j}}{\sum_{j=0}^{\infty} l_{j}-\sum_{j=0}^{x-1} l_{j}}=\frac{\sum_{j=x+1}^{\infty} l_{j}}{\sum_{j=x}^{\infty} l_{j}}=1-\frac{l_{x}}{\sum_{j=x}^{\infty} l_{j}} .
$$

Parameter estimates for $\alpha, \beta$, and $p$ were found using maximum likelihood techniques (Hilborn and Mangel 1997). In addition, we used maximum likelihood to estimate $\delta$, an additional, additive probability of crickets immigrating and/or dying the first day after release (above and beyond losses from normal mortality and senescence). Models are included in a Mathematica notebook that is available for download in the Supplement. A stepwise procedure was used to construct models, with sex-specific parameters and a non-senescent model $(\beta=0)$ being considered at each step. Akaike's Information Criterion (AIC) was used to select among the models. When the difference in AIC between two models $\left(\Delta_{i}\right)$ is less than 2 , there is no statistical basis for choosing among them (Burnham and Anderson 2002). When $\Delta_{i}>2$ one can dismiss models with the higher AIC values as having significantly less support. Sex-specific median and maximum life span for males and females was calculated from model C (see Results), where median life span is defined as the estimated age at which half of the individuals had died (Finch and Pike 1996) and maximum life span is the age at which $99 \%$ of individuals have died.

\section{RESUlts}

The three best-fit models (Table 1, models A, B, C) all have substantial support $\left(\Delta_{i}<2\right)$. These models all include sex-specific observation probabilities $(p)$, sexspecific mortality rates $(\alpha)$, and a non-sex-specific senescence term ( $\beta$ ) (Fig. 1). All these models also include a non-sex-specific parameter $(\delta)$ describing loss of individuals due to immigration or death immediately after release. Thus, in this wild cricket population, males have higher mortality rates throughout life, but the sexes appear to age at similar rates.

Of the three best-fit models, $\mathrm{A}$ and $\mathrm{B}$ include parameters that explain variation in extrinsic factors that change from day to day. These parameters provide some improvement in fit over model $\mathrm{C}$, which includes only sex-specific mortality rates and capture probabilities, a non-sex-specific senescence term, and $\delta$. However, the biological significance of the 70 day-specific recapture rate parameters included in model $\mathrm{A}$ is questionable. Considering models that include temperaturedependent recapture rates (models $\mathrm{B}, \mathrm{E}, \mathrm{J}$; for temperature plot see Fig. 2), model B with equal senescence rates in both sexes is a better fit than the model that includes sex-specific senescence $(\mathrm{E})$ and the model that does not include senescence (J). Similarly, considering models without temperature dependence (models $\mathrm{C}, \mathrm{G}$, I), model $\mathrm{C}$ with equal senescence rates in both sexes is a better fit than the model that includes sex-specific senescence $(G)$ and the model that does not include senescence (I). We conclude that senescence is occurring, but that it is not sex specific. Parameter estimates based on model B are $\tau=-0.03, p_{\mathrm{m}}=0.0014, p_{\mathrm{f}}=0.0024, \alpha_{\mathrm{m}}=$ $0.038, \alpha_{\mathrm{f}}=0.025, \delta=0.26, \beta=0.023$ (subscripts $\mathrm{m}$ and $\mathrm{f}$ refer to male and female). While a classical statistical 
TABLE 1. Summary of models for black field crickets (Teleogryllus commodus), ordered with the best-fit model at the top.

\begin{tabular}{|c|c|c|c|c|c|c|c|c|}
\hline Model name & & & aramete & & & $n$ & $\Delta_{i}$ & $w_{i}$ \\
\hline A) Senescence, day- + sex-specific recapture & & $p_{\text {day }}+p_{\text {sex }}$ & $\alpha_{\mathrm{m}}, \alpha_{\mathrm{f}}$ & $\delta$ & $\beta$ & 76 & 0.0 & 0.34 \\
\hline B) Senescence with temperature-dependent recapture & $\tau$ & $p_{\mathrm{m}}, p_{\mathrm{f}}$ & $\alpha_{\mathrm{m}}, \alpha_{\mathrm{f}}$ & $\delta$ & $\beta$ & 7 & 0.94 & 0.21 \\
\hline C) Senescence & & $p_{\mathrm{m}}, p_{\mathrm{f}}$ & $\alpha_{\mathrm{m}}, \alpha_{\mathrm{f}}$ & $\delta$ & $\beta$ & 6 & 1.6 & 0.15 \\
\hline $\begin{array}{l}\text { D) Sex-specific senescence, without sex-specific background } \\
\text { mortality }\end{array}$ & & $p_{\mathrm{m}}, p_{\mathrm{f}}$ & $\alpha$ & $\delta$ & $\beta_{\mathrm{m}}, \beta_{\mathrm{f}}$ & 6 & 2.4 & 0.10 \\
\hline $\begin{array}{l}\text { E) Sex-specific senescence with temperature-dependent } \\
\text { recapture }\end{array}$ & $\tau$ & $p_{\mathrm{m}}, p_{\mathrm{f}}$ & $\alpha_{\mathrm{m}}, \alpha_{\mathrm{f}}$ & $\delta$ & $\beta_{\mathrm{m}}, \beta_{\mathrm{f}}$ & 8 & 2.9 & 0.08 \\
\hline F) Sex-specific initial loss & & $p_{\mathrm{m}}, p_{\mathrm{f}}$ & $\alpha_{\mathrm{m}}, \alpha_{\mathrm{f}}$ & $\delta_{\mathrm{m}}, \delta_{\mathrm{f}}$ & $\beta$ & 7 & 3.5 & 0.06 \\
\hline G) Sex-specific senescence & & $p_{\mathrm{m}}, p_{\mathrm{f}}$ & $\alpha_{\mathrm{m}}, \alpha_{\mathrm{f}}$ & $\delta$ & $\beta_{\mathrm{m}}, \beta_{\mathrm{f}}$ & 7 & 3.6 & 0.06 \\
\hline H) Senescence with sex-specific recapture & & $p_{\mathrm{m}}, p_{\mathrm{f}}$ & $\alpha$ & $\delta$ & $\beta$ & 5 & 9.9 & $<0.01$ \\
\hline I) Sex-specific recapture & & $p_{\mathrm{m}}, p_{\mathrm{f}}$ & $\alpha_{\mathrm{m}}, \alpha_{\mathrm{f}}$ & $\delta$ & & 5 & 17.7 & $<0.01$ \\
\hline J) No senescence with temperature-dependent recapture & $\tau$ & $p_{\mathrm{m}}, p_{\mathrm{f}}$ & $\alpha_{m}, \alpha_{f}$ & $\delta$ & & 6 & 17.9 & $<0.01$ \\
\hline K) Sex-specific mortality & & $p$ & $\alpha_{\mathrm{m}}, \alpha_{\mathrm{f}}$ & $\delta$ & & 4 & 53.6 & $<0.01$ \\
\hline L) With initial loss & & $p$ & $\alpha$ & $\delta$ & & 3 & 86.4 & $<0.01$ \\
\hline M) Constant mortality & & $p$ & $\alpha$ & & & 2 & 158 & $<0.01$ \\
\hline
\end{tabular}

Notes: Models include temperature dependence $(\tau)$, sex-specific observation probabilities $(p)$, sex-specific mortality rates $(\alpha)$, a non-sex-specific senescence term $(\beta)$, and a non-sex-specific parameter $(\delta)$ describing loss of individuals due to immigration or death immediately after release. Subscripts $\mathrm{m}$ and $\mathrm{f}$ refer to sex-specific variables for males and females. The number of parameters fitted in each model is given as $n ; \Delta_{i}$ is the difference in AIC between model $i$ and the model with the lowest AIC (model A); and Akaike weights are given as $w_{i}$ (Burnham and Anderson 2002).

framework typically would not test for sex-specific $\beta$ in the absence of sex-specific $\alpha$, we include model $\mathrm{D}$ for completeness.

The model fit in Fig. 1 cannot be visualized in relation to the actual daily mortality rate data because dates of birth are only known distributionally for the entire population and dates of death are only known probabilistically for each individual. However, a plot of captures per day after first release can be used to visualize raw data and model predictions (Fig. 3). Characteristically, the differences between predictions based on senescent models and non-senescent models are slight in this type of plot.

Based on parameter estimates from the Gompertz senescence model (Model C), the median life span was calculated to be 15.2 days for male crickets and 21.4 days for female crickets. The predicted maximum life

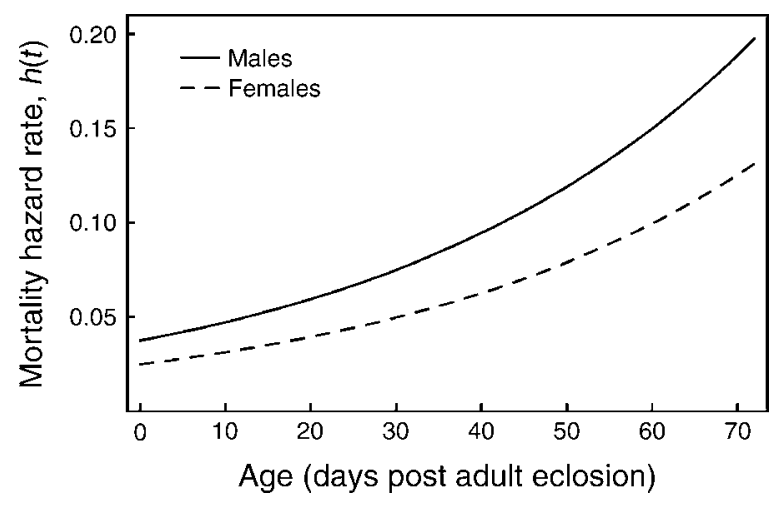

FIG. 1. Increasing mortality hazard rate, $h(t)$, of male (solid line) and female (dashed line) black field crickets (Teleogryllus commodus) with age. Model B is shown (see Table 1). The hazard rate $h(t)$ is the continuous form of the daily mortality rate $m(x)$, where $x$ is age. span was 57.9 days for males and 72.0 days for females. This difference in life expectancy is due to the significant difference in background mortality $(\alpha)$ between the sexes.

\section{DisCusSION}

We estimated and compared sex-dependent mortality and aging rates in a natural population of a species that is relatively short lived and difficult to track. This was made possible by the use of a new, unified method for analyzing capture-mark-recapture data where exact dates of birth and death are unknown. Demographic senescence occurred in both male and female $T$. commodus. Males experienced higher mortality rates than females, but the rate of senescence did not differ between the sexes. Females were estimated to live 6.2 days longer than males, on average. To our knowledge, this is the first study to provide compelling evidence of senescence and sex-specific mortality in both sexes in a natural population of a short-lived invertebrate species when the dates of birth and death are unknown.

Despite the striking patterns of sex-specific longevity in a variety of organisms, the underlying causes are far from resolved (Owens 2002, Clutton-Brock and Isvaran 2007, Bonduriansky et al. 2008). As we found in the present study, males commonly show higher mortality rates throughout life than females, both in laboratory studies of insects (Promislow et al. 1996, Pletcher et al. 2000, Maklakov et al. 2006, Styler et al. 2007) and in natural populations of large mammals (Loison et al. 1999, Gaillard et al. 2004). There are two possible explanations that can explain this pattern. First, higher mortality rates in males can be mainly due to extrinsic effects that are currently present in the population, such as predation, parasitism, and accidents, given that male reproductive strategies are predicted to involve greater risk-taking (sacrificing of survival for reproductive opportunities) 


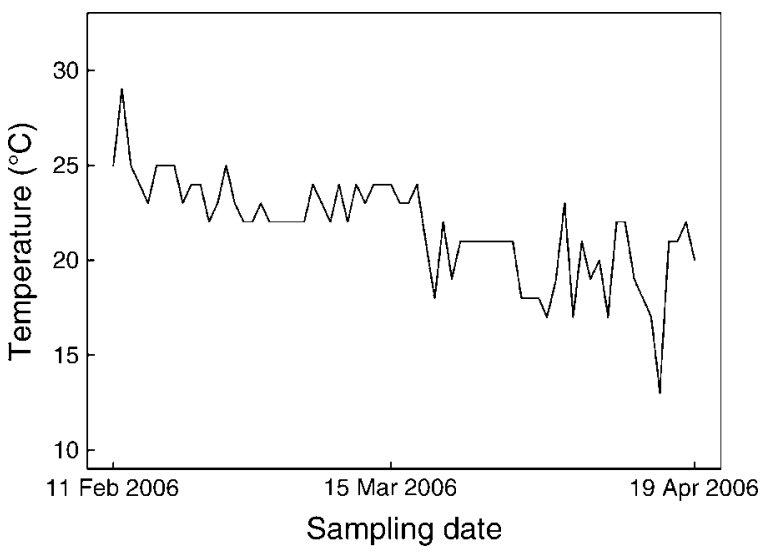

FIG. 2. Nightly ambient temperatures during the sampling period, measured between 24:00 and 01:00 hours each day, continuously from 11 February to 19 April.

than female reproductive strategies (Bonduriansky et al. 2008). For male crickets, predation by phonotactic predators and infestation by parasitoids are very likely to be higher than for females, due to the conspicuousness of male advertisement calls (reviewed in Zuk and Kolluru 1998). Bright coloration in guppies (Godin and McDonough 2003) that acts as a visual sexual signal, seismic sexual signaling in wolf spiders (Roberts et al. 2007), and larger male body size in kudu (Owen-Smith 1993) are
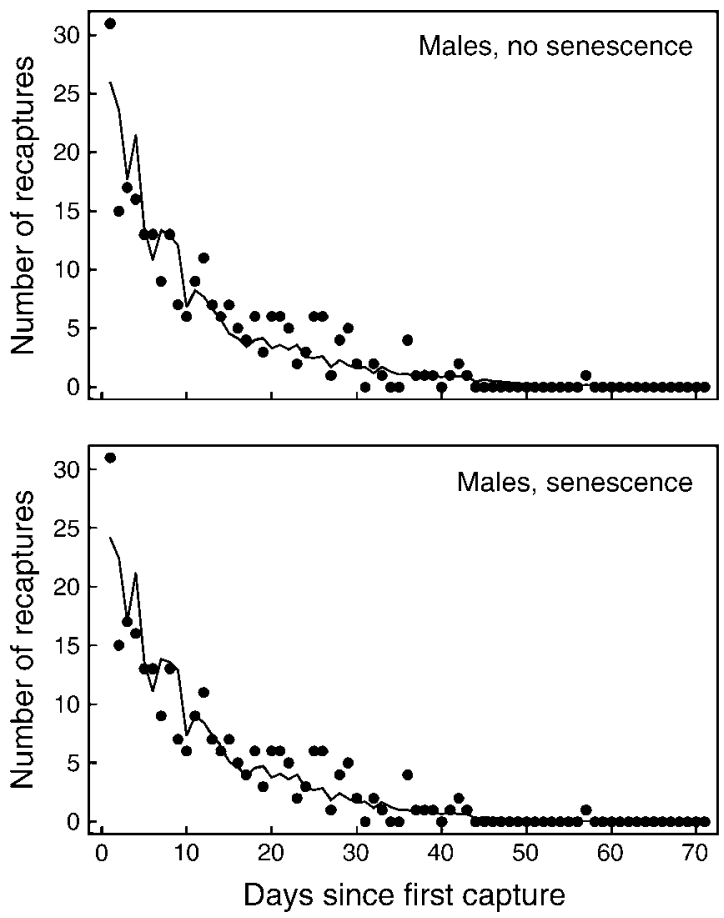

other examples of sexually selected traits that increase extrinsic mortality rates in males.

Second, higher mortality rates in males can have intrinsic causes that have evolved through differences in the strength of sexual selection on females and males, with competitive mating ability often being much more strongly selected in males (Trivers 1972, Kruger and Nesse 2006, Bonduriansky et al. 2008). Sexually selected male traits that impose elevated intrinsic costs on males include ornamental color patterns (Brooks 2000), weapons (Emlen and Nijhout 2000), larger male body size (Owen-Smith 1993, Carranza and Pérez-Barbería 2007), and elevated energetic expenditure on sexual display (Kotiaho et al. 1998). Many intrinsic costs associated with sexual selection are mediated by male hormone levels (Salvador et al. 1996, Alonso-Alvarez et al. 2007).

These two possible reasons for the bias toward overall higher male mortality rates are not entirely independent of each other, as intrinsic causes are supposed to have been shaped by extrinsic effects on an evolutionary timescale. A factor that might affect both cases is the possibility of condition dependence of male performance traits that can counterbalance the negative effect on survival to a certain extent, as shown in guppies (Reznick et al. 2004) and wolf spiders (Lindström et al. 2006).

If males emerged into adulthood earlier in the season than females, males could therefore have died earlier in
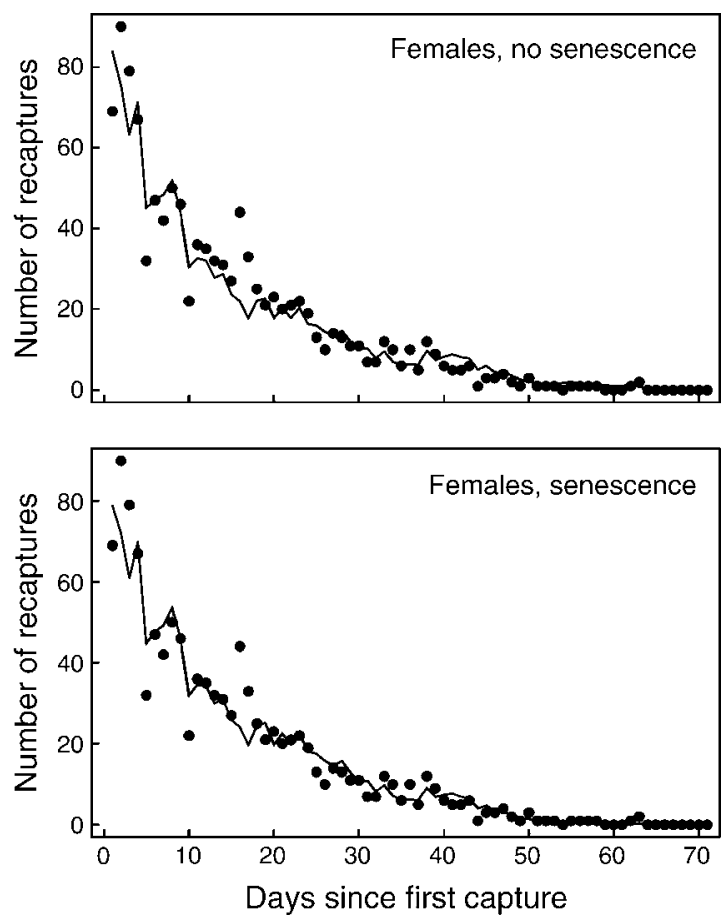

FIG. 3. The actual number of recaptures for each sex is plotted as solid dots. The predicted number of recaptures is plotted as solid lines. The predicted number is adjusted for person-minutes of observation and is either a constant mortality rate in the "no senescence" plots or an increasing rate of mortality with age in the "senescence" plots. Predictions for the top (no senescence) panels are based on model I; predictions for the bottom (senescence) panels are based on model B. In this type of plot, there are usually only slight differences between predictions based on senescent vs. non-senescent models. 
our marked cohort, causing the estimated difference in median life span of 6.2 days between males and females. However, we did not find a difference in the time until eclosion to adulthood between male and female nymphs caught in the first two weeks of the present study $(N=84$ males, $N=146$ females; Mann-Whitney $U$ test, $P=0.12$ ) or in crickets raised in field enclosures (F. Zajitschek, R. Bonduriansky, S. R. K. Zajitschek, and R. C. Brooks, unpublished data). Moreover, results from a large-scale laboratory-based experiment showed that there was a significant difference in male and female development time $(N=1417$ males, $N=1724$ females; $t=3.18, P<$ 0.01 ), but the difference was only $1 \mathrm{day}$, and the effect was in the opposite direction, i.e., females eclosed earlier than males (Zajitschek et al. 2007). Together, these results suggest that sex differences in the timing of adult eclosion are unlikely to account for the estimated difference in life span between the sexes.

Contrary to expectations, senescence rate did not differ between males and females. There are several possible explanations for this observation. First, with few deaths occurring at early and at late ages, the power to detect differences in point estimates of true mortality rates can be limited. This problem can be ameliorated by using maximum likelihood techniques to estimate the "rate of aging" parameter in the Gompertz model (Promislow et al. 1999), which is the approach we used in the present analysis. Our failure to detect sex-specific senescence rates suggests that demographic aging is not sex dependent and, therefore, that it is not strongly influenced by the profoundly different schedules of reproductive effort documented in male and female $T$. commodus (Hunt et al. 2006, Maklakov et al. 2008). This finding bears further direct investigation because it is at odds with a large body of general evidence for differences in male and female reproductive effort and survival costs of reproduction (Roff 1992, Stearns 1992, Reznick et al. 2000). Sex differences in senescence remain poorly characterized, and their causes are poorly understood (Bonduriansky et al. 2008). Senescence does not, however, only affect mortality rates, but all aspects of organismal performance, and a comprehensive measure of senescence rate reflects the product of survival and reproduction (Partridge and Barton 1993, 1996). This means that it is theoretically possible for sexspecific senescence to occur in male and female reproductive schedules, even if there is no difference in the senescent increase in mortality rates.

It has been suggested previously that senescence in the wild may be hard to observe because not many individuals in a population will live to an age for demographic aging to be detectable (Kirkwood and Austad 2000). Senescent patterns in age-specific survival in the wild have been shown, although mostly in relatively long-lived taxa such as mammals (Promislow 1991, Jorgenson et al. 1997, Loison et al. 1999, Beauplet et al. 2006) and birds (Newton and Rothery 1997, Sanz and Moreno 2000, Holmes et al. 2001, Orell and Belda
2002). The extent to which senescence is detectable in wild populations of animals (such as insects, nematodes, and rodents) that are commonly used in laboratory studies of aging has been an open question for some time (Comfort 1956, Hayflick 2000). This is thought to be for a variety of analytic and logistic reasons.

Statistical methods that were especially developed to model capture-mark-recapture data (CJS method) have long made it possible to study senescence in the wild when the time of birth can be specified with useful precision (Nichols et al. 1997, Loison et al. 1999, Chaloupka and Limpus 2005), but the time of death is known only probabilistically from recapture or resighting data. Models of aging, such as the Gompertz model that we use in the present study, have also been fitted to capture-mark-recapture data in order to test for senescence (Gaillard et al. 2004). However, by combining these previously available methods with the a new life-table analysis developed by Müller et al. (2004), we were able, for the first time, to use data from capturemark-recapture studies in which neither the dates of birth nor death of experimental animals were known, and to use our estimates to test for sex-dependent mortality and senescence.

In addition to the statistical challenges, an equally important cause of the paucity of insect aging studies in the wild might be an issue of body size of the study species and thus the tractability of marking, resighting, or recapturing animals. Estimating age-dependent mortality rates with a high enough temporal resolution to subsequently test for aging in short-lived insects has been shown to be possible for male antler flies (Protopiophila litigata), which spend their whole adult life in the very restricted geographical area of a discarded moose or deer antler (Bonduriansky and Brassil 2002), and neriid flies (Telostylinus angusticollis) of both sexes, which aggregate on tree trunks (Kawasaki et al. 2008). Not all insect species aggregate in small geographical areas. Nevertheless, other insect species have been used before in capture-mark-recapture studies in the wild, but these studies generally yielded insufficient data to test comprehensively for sex-specific demographic aging (e.g., milkweed beetles, McCauley [1983]; damselflies, Hafernik and Garrison [1986]). The method that we apply here will facilitate research on other species where dates of both birth and death are difficult to estimate in the natural environment. Because most of the major model species used in laboratory studies of aging belong in this group, we hope that our approach will enhance the ability of various research programs to combine the idiosyncratic advantages of studies in the wild and in the laboratory and thus provide a more complete and meaningful understanding of the evolution of aging (sensu Bartholomew 1986, Greene 2005).

Our analyses are based on several assumptions. For example, marked crickets are assumed not to disperse or diffuse out of the sampling area, and not to lose their marks. If these assumptions are violated, our estimated 
mortality rates and estimates of age-dependent increase in mortality would be inflated, as the probability that either process occurs may increase with time after release. We are confident, however, that diffusion and marker loss had only a minor, if any, effect. Recapture rate of marked crickets outside the regular sampling area was extremely low, despite considerable sampling effort. A previous study also observed male $T$. commodus calling from the same position for up to 14 days, showing that they appear to stay in the same area for extended periods (Campbell 1977). In our pilot study on a sample of 25 marked crickets maintained under simulated natural conditions in the laboratory, no loss of marks occurred over 30 days, suggesting that marked crickets are unlikely to have lost their marks in the field.

Our method also assumes that the wild population that is studied has to have a stable and stationary age structure. A stationary population is a special case of a stable population, in which births balance the deaths; i.e., the population does not change in size. Seasonal effects (e.g., environment conditions such as temperature), have been shown to affect population dynamics in many species (e.g., Coulson et al. 2001). Thus, an environment-dependent increase in mortality rate, or decrease in recapture probability, throughout the study period could be confounding the estimated senescent increase in age-dependent mortality rate. However, there are no obvious environmental or ecological factors that could account for our results. For example, although ambient night temperature decreased over the study period, inclusion of temperature-dependent recapture in our model had only a small effect on estimates of agedependent mortality rates. We did not observe any change in population density or resource availability (e.g., grass, seeds) over the duration of our study. Including day-specific capture rates improved model fit, but the general biological interpretation of the 70 dayspecific parameters included in this model is obscure. To test quantitatively for signatures of environmental effects on resighting probability, we compared the daily resighting probabilities of the first and last $20 \%$ of crickets entering the study. No difference between these cohorts in resighting probability was observed either in males $(t=0.60, \mathrm{df}=62, P=0.55)$ or in females $(t=1.68$, $\mathrm{df}=196, P=0.09)$. Temporal changes in unmeasured ambient parameters are, in any case, unlikely to have had substantial effects on our estimates of age-dependent mortality rates because the marked animals represent a nonsynchronous cohort. This means that any environmental changes over the study period would not affect all marked animals at the same age (e.g., a seasonal increase in mortality rate late in the experiment would have affected young as well as old animals). Thus, temporal changes in ambient conditions affecting resighting rates are unlikely to have confounded our estimates of life expectancy or aging.

In conclusion, we have shown that it is possible to test for demographic senescence in wild, hard-to-track animals when dates of birth and death are not known, using a new, flexible methodological framework that can be individually adapted to related questions and that should contribute to a substantial advance of research in this field. We were able to detect demographic senescence in both male and female crickets, with males having higher overall mortality rates and a shorter life span than females. However, the senescent increase in mortality rates was not different between the sexes. These findings accord with laboratory-based empirical findings and theoretical suggestions that males should experience higher extrinsic death hazards in the wild, although we found no evidence that these differences in mortality rate are associated with sex-specific rates of demographic aging. Characterizing sex-specific mortality rates in natural populations of a wider range of species, notably invertebrates, will enhance the interpretation of results from laboratory studies. Similarly, new insights about patterns of mortality and aging in the wild can serve to generate new hypotheses that can be tested under laboratory conditions, and will ultimately shed light on the complex interaction between sexspecific selection, extrinsic mortality, and senescence.

\section{ACKNOWLEDGMENTS}

We thank Susanne Zajitschek, Misha Donohoe, Matt Hall, Barbara Tschirren, and Erik Postma for their help in the field. The project was supported by an ARC Discovery grant and separate ARC fellowships to R. C. Brooks and R. Bonduriansky.

\section{Literature Cited}

Abrams, P. A., and D. Ludwig. 1995. Optimality theory, Gompertz' law, and the disposable soma theory of senescence. Evolution 49:1055-1066.

Alonso-Alvarez, C., S. Bertrand, B. Faivre, O. Chastel, and G. Sorci. 2007. Testosterone and oxidative stress: the oxidation handicap hypothesis. Proceedings of the Royal Society B 274: 819-825.

Arnqvist, G., and L. Rowe. 2005. Sexual conflict. Princeton University Press, Princeton, New Jersey, USA.

Bartholomew, G. A. 1986. The role of natural-history in contemporary biology. BioScience 36:324-329.

Beauplet, G., C. Barbraud, W. Dabin, C. Kussener, and C. Guinet. 2006. Age-specific survival and reproductive performances in fur seals: evidence of senescence and individual quality. Oikos 112:430-441.

Bonduriansky, R., and C. E. Brassil. 2002. Rapid and costly ageing in wild male flies. Nature 420:377.

Bonduriansky, R., A. Maklakov, F. Zajitschek, and R. Brooks. 2008. Sexual selection, sexual conflict and the evolution of ageing and lifespan. Functional Ecology 22:443-453.

Bronikowski, A. M., and D. E. L. Promislow. 2005. Testing evolutionary theories of aging in wild populations. Trends in Ecology and Evolution 20:271-273.

Brooks, R. 2000. Negative genetic correlation between male sexual attractiveness and survival. Nature 406:67-70.

Browning, T. O. 1954. Observations on the ecology of the Australian field cricket, Gryllulus Commodus Walker, in the field. Australian Journal of Zoology 2:205-222.

Burnham, K. P., and D. R. Anderson. 2002. Model selection and multimodel inference: a practical information-theoretic approach. Springer, New York, New York, USA.

Campbell, D. J. 1977. Migratory and social behaviour affecting spatial pattern in populations of the field cricket Teleogryllus 
commodus (Walker). Dissertation. University of New South Wales. Sydney, Australia.

Carey, J. R. 2001. Insect biodemography. Annual Review of Entomology 46:79-110.

Carnes, B. A., and S. J. Olshansky. 1997. A biologically motivated partitioning of mortality. Experimental Gerontology 32:615-631.

Carranza, J., S. Alarcos, C. B. Sanchez-Prieto, J. Valencia, and C. Mateos. 2004. Disposable-soma senescence mediated by sexual selection in an ungulate. Nature 432:215-218.

Carranza, J., and F. J. Pérez-Barbería. 2007. Sexual selection and senescence: Male size-dimorphic ungulates evolved relatively smaller molars than females. American Naturalist 170:370-380.

Chaloupka, M., and C. Limpus. 2005. Estimates of sex- and age-class-specific survival probabilities for a southern Great Barrier Reef green sea turtle population. Marine Biology 146: 1251-1261.

Charlesworth, B. 1994. Evolution in age-structured populations. Second edition. Cambridge University Press, New York, New York, USA.

Clutton-Brock, T., and K. Isvaran. 2007. Sex differences in ageing in natural populations of vertebrates. Proceedings of the Royal Society B 274:3097-3104.

Comfort, A. 1956. The biology of senescence. Elsevier North Holland, New York, New York, USA.

Cormack, R. M. 1964. Estimates of survival from sighting of marked animals. Biometrika 51:429-438.

Coulson, T., E. A. Catchpole, S. D. Albon, B. J. T. Morgan, J. M. Pemberton, T. H. Clutton-Brock, M. J. Crawley, and B. T. Grenfell. 2001. Age, sex, density, winter weather, and population crashes in Soay sheep. Science 292:1528-1531.

Crespi, B. J. 1988. Risks and benefits of lethal male fighting in the colonial, polygynous thrips Hoplothrips karnyi (Insecta: Thysanoptera). Behavioral Ecology and Sociobiology 22: 293-301.

Crespin, L., M. P. Harris, J.-D. Lebreton, and S. Wanless. 2006. Increased adult mortality and reduced breeding success with age in a population of common guillemot Uria aalge using marked birds of unknown age. Journal of Avian Biology 37:273-282.

Emlen, D. J., and H. F. Nijhout. 2000. The development and evolution of exaggerated morphologies in insects. Annual Review of Entomology 45:661-708.

Endler, J. A. 1980. Natural selection on color patterns in Poecilia reticulata. Evolution 34:76-91.

Finch, C. E. 1994. Longevity, senescence, and the genome. University of Chicago Press, Chicago, Illinois, USA.

Finch, C. E., and M. C. Pike. 1996. Maximum life span predictions from the Gompertz mortality model. Journals of Gerontology Series A 51:B183-B194.

Fox, C. W., L. Dublin, and S. J. Pollitt. 2003. Gender differences in lifespan and mortality rates in two seed beetle species. Functional Ecology 17:619-626.

Gaillard, J. M., A. Viallefont, A. Loison, and M. Festa-Bianchet. 2004. Assessing senescence patterns in populations of large mammals. Animal Biodiversity and Conservation 27:47-58.

Godin, J. G., and H. E. McDonough. 2003. Predator preference for brightly colored males in the guppy: a viability cost for a sexually selected trait. Behavioral Ecology 14:194-200.

Gompertz, B. 1825. On the nature of the function expressive of the law of human mortality, and on a new mode of determining the value of life contingencies. Philosophical Transactions of the Royal Society of London 115.

Graves, B. M. 2007. Sexual selection effects on the evolution of senescence. Evolutionary Ecology 21:663-668.

Greene, H. W. 2005. Organisms in nature as a central focus for biology. Trends in Ecology and Evolution 20:23-27.

Hafernik, J. E., and R. W. Garrison. 1986. Mating success and survival rate in a population of damselflies: results at variance with theory. American Naturalist 128:353-365.
Hamilton, W. D. 1966. Moulding of senescence by natural selection. Journal of Theoretical Biology 12:12-45.

Hayflick, L. 2000. The future of ageing. Nature 408:267-269.

Hilborn, R., and M. Mangel. 1997. The ecological detective: confronting models with data. Princeton University Press, Princeton, New Jersey, USA.

Hogan, T. W. 1966. Physiological differences between races of Teleogryllus commodus (Walker) (Orthoptera: Gryllidae) related to a proposed genetic approach to control. Australian Journal of Zoology245-251.

Holmes, D. J., R. Fluckiger, and S. N. Austad. 2001. Comparative biology of aging in birds: an update. Experimental Gerontology 36:869-883.

Hunt, J., R. Brooks, M. D. Jennions, M. J. Smith, C. L. Bentsen, and L. F. Bussière. 2004. High-quality male field crickets invest heavily in sexual display but die young. Nature 432:1024-1027.

Hunt, J., M. D. Jennions, N. Spyrou, and R. Brooks. 2006. Artificial selection on male longevity influences age-dependent reproductive effort in the black field cricket, Teleogryllus commodus. American Naturalist 168:E72-E86.

Jolly, G. M. 1965. Explicit estimates from capture-recapture data with both death and immigration - stochastic model. Biometrika 52:225-247.

Jorgenson, J. T., M. Festa-Bianchet, J. M. Gaillard, and W. D. Wishart. 1997. Effects of age, sex, disease, and density on survival of bighorn sheep. Ecology 78:1019-1032.

Kawasaki, N., C. E. Brassil, R. Brooks, and R. Bonduriansky. 2008. Environmental effects on the expression of lifespan and aging: an extreme contrast between wild and captive cohorts of Telostylinus angusticollis (Diptera: Neriidae). American Naturalist 172:346-357.

Kirkwood, T. B. L., and S. N. Austad. 2000. Why do we age? Nature 408:233-238.

Kirkwood, T. B. L., and M. R. Rose. 1991. Evolution of senescence: late survival sacrificed for reproduction. Philosophical Transactions of the Royal Society B 332:15-24.

Kotiaho, J. S., R. V. Alatalo, J. Mappes, M. G. Nielsen, S. Parri, and A. Rivero. 1998. Energetic costs of size and sexual signalling in a wolf spider. Proceedings of the Royal Society of London Series B 265:2203-2209.

Kruger, D. J., and R. M. Nesse. 2006. An evolutionary lifehistory framework for understanding sex differences in human mortality rates. Human Nature: An Interdisciplinary Biosocial Perspective 17:74-97.

Lebreton, J.-D., K. P. Burnham, J. Clobert, and D. R. Anderson. 1992. Modeling survival and testing biological hypotheses using marked animals: a unified approach with case-studies. Ecological Monographs 62:67-118.

Liker, A., and T. Szekely. 2005. Mortality costs of sexual selection and parental care in natural populations of birds. Evolution 59:890-897.

Lindström, L., J. J. Ahtiainen, J. Mappes, J. S. Kotiaho, A. Lyytinen, and R. V. Alatalo. 2006. Negatively condition dependent predation cost of a positively condition dependent sexual signalling. Journal of Evolutionary Biology 19:649656.

Loison, A., M. Festa-Bianchet, J. M. Gaillard, J. T. Jorgenson, and J. M. Jullien. 1999. Age-specific survival in five populations of ungulates: evidence of senescence. Ecology 80:2539-2554.

Magwere, T., T. Chapman, and L. Partridge. 2004. Sex differences in the effect of dietary restriction on life span and mortality rates in female and male Drosophila melanogaster. Journals of Gerontology Series A 59:3-9.

Maklakov, A. A., U. Friberg, D. K. Dowling, and G. Arnqvist. 2006. Within-population variation in cytoplasmic genes affects female life span and aging in Drosophila melanogaster. Evolution 60:2081-2086.

Maklakov, A. A., S. J. Simpson, F. Zajitschek, M. D. Hall, J. Dessmann, F. Clissold, D. Raubenheimer, R. Bonduriansky, 
and R. C. Brooks. 2008. Sex-specific fitness effects of nutrient intake on reproduction and lifespan. Current Biology 18: 1062-1066.

McCauley, D. E. 1983. An estimate of the relative opportunities for natural and sexual selection in a population of milkweed beetles. Evolution 37:701-707.

McDonald, D. B., J. W. Fitzpatrick, and G. E. Woolfenden. 1996. Actuarial senescence and demographic heterogeneity in the Florida Scrub Jay. Ecology 77:2373-2381.

Medawar, P. B. 1952. An unsolved problem of biology. H. K. Lewis, London, UK.

Müller, H. G., J. L. Wang, J. R. Carey, E. P. Caswell-Chen, C. Chen, N. Papadopoulos, and F. Yao. 2004. Demographic window to aging in the wild: Constructing life tables and estimating survival functions from marked individuals of unknown age. Aging Cell 3:125-131.

Mysterud, A., N. G. Yoccoz, N. C. Stenseth, and R. Langvatn. 2001. Effects of age, sex and density on body weight of Norwegian red deer: evidence of density-dependent senescence. Proceedings of the Royal Society B 268:911-919.

Newton, I., and P. Rothery. 1997. Senescence and reproductive value in Sparrowhawks. Ecology 78:1000-1008.

Nichols, J. D., J. E. Hines, and P. Blums. 1997. Tests for senescent decline in annual survival probabilities of Common Pochards, Aythya ferina. Ecology 78:1009-1018.

Nussey, D. H., L. E. B. Kruuk, A. Donald, M. Fowlie, and T. H. Clutton-Brock. 2006. The rate of senescence in maternal performance increases with early-life fecundity in red deer. Ecology Letters 9:1342-1350.

Orell, M., and E. J. Belda. 2002. Delayed cost of reproduction and senescence in the willow tit Parus montanus. Journal of Animal Ecology 71:55-64.

Owen-Smith, N. 1993. Comparative mortality rates of male and female kudus: the costs of sexual size dimorphism. Journal of Animal Ecology 62:428-440.

Owens, F. 2002. Sex differences in mortality rate. Science 297: 2008-2009.

Parker, G. A. 1979. Sexual selection and sexual conflict. Pages 123-166 in N. S. Blum and N. A. Blum, editors. Sexual selection and reproductive competition in insects. Academic Press, London, UK.

Partridge, L., and N. H. Barton. 1993. Optimality, mutation and the evolution of ageing. Nature 362:305-311.

Partridge, L., and N. H. Barton. 1996. On measuring the rate of ageing. Proceedings of the Royal Society B 263:1365-1371.

Pletcher, S. D., A. A. Khazaeli, and J. W. Curtsinger. 2000. Why do life spans differ? Partitioning mean longevity differences in terms of age-specific mortality parameters. Journals of Gerontology Series A 55:B381-B389.

Pollock, K. H. 1991. Modeling capture, recapture, and removal statistics for estimation of demographic parameters for fish and wildlife populations: past, present, and future. Journal of the American Statistical Association 86:225-238.

Promislow, D. E. L. 1991. Senescence in natural populations of mammals: a comparative study. Evolution 45:1869-1887.

Promislow, D. 2003. Mate choice, sexual conflict, and evolution of senescence. Behavior Genetics 33:191-201.

Promislow, D. E. L., and P. H. Harvey. 1990. Living fast and dying young: A comparative analysis of life-history variation among mammals. Journal of Zoology 220:417-437.

Promislow, D. E. L., M. Tatar, A. A. Khazaeli, and J. W. Curtsinger. 1996. Age-specific patterns of genetic variance in Drosophila melanogaster. 1. Mortality. Genetics 143:839-848.

Promislow, D. E. L., M. Tatar, S. Pletcher, and J. R. Carey. 1999. Below-threshold mortality: implications for studies in evolution, ecology and demography. Journal of Evolutionary Biology 12:314-328.

Reid, J. M., E. M. Bignal, S. Bignal, D. I. McCracken, and P. Monaghan. 2003. Age-specific reproductive performance in red-billed choughs Pyrrhocorax pyrrhocorax: patterns and processes in a natural population. Journal of Animal Ecology 72:765-776.

Reznick, D. N., M. J. Bryant, D. Roff, C. K. Ghalambor, and D. E. Ghalambor. 2004. Effect of extrinsic mortality on the evolution of senescence in guppies. Nature 431:1095-1099.

Reznick, D., G. Buckwalter, J. Groff, and D. Elder. 2001. The evolution of senescence in natural populations of guppies (Poecilia reticulata): a comparative approach. Experimental Gerontology 36:791-812.

Reznick, D., L. Nunney, and A. Tessier. 2000. Big houses, big cars, superfleas and the costs of reproduction. Trends in Ecology and Evolution 15:421-425.

Ricklefs, R. E. 2000. Intrinsic aging-related mortality in birds. Journal of Avian Biology 31:103-111.

Roberts, J. A., P. W. Taylor, and G. W. Uetz. 2007. Consequences of complex signaling: predator detection of multimodal cues. Behavioral Ecology 18:236-240.

Roff, D. A. 1992. The evolution of life histories: theory and analysis. Chapman and Hall, New York, New York, USA.

Salvador, A., J. P. Veiga, J. Martin, P. Lopez, M. Abelenda, and M. Puerta. 1996. The cost of producing a sexual signal: Testosterone increases the susceptibility of male lizards to ectoparasitic infestation. Behavioral Ecology 7:145-150.

Sanz, J. J., and J. Moreno. 2000. Delayed senescence in a southern population of the pied flycatcher (Ficedula hypoleuca). Ecoscience 7:25-31.

Seber, G. A. F. 1965. A note on multiple-recapture census. Biometrika 52:249-259.

Stearns, S. C. 1992. The evolution of life histories. Oxford University Press, Oxford, UK.

Styler, L. M., J. R. Carey, J. L. Wang, and T. W. Scott. 2007. Mosquitos do senesce: departure from the paradigm of constant mortality. American Journal of Tropical Medicine and Hygiene 76:111-117.

Svensson, E., and B. C. Sheldon. 1998. The social context of life history evolution. Oikos 83:466-477.

Toigo, C., J. M. Gaillard, M. Festa-Bianchet, E. Largo, J. Michallet, and D. Maillard. 2007. Sex- and age-specific survival of the highly dimorphic Alpine ibex: evidence for a conservative life-history tactic. Journal of Animal Ecology 76:679-686.

Trivers, R. L. 1972. Parental investment and sexual selection. Pages 136-179 in B. Campbell, editor. Sexual selection and the descent of man, 1871-1971. Aldine-Atherton, Chicago, Illinois, USA.

Williams, G. C. 1957. Pleiotropy, natural selection, and the evolution of senescence. Evolution 11:398-411.

Williams, P. D., and T. Day. 2003. Antagonistic pleiotropy, mortality source interactions, and the evolutionary theory of senescence. Evolution 57:1478-1488.

Williams, P. D., T. Day, Q. Fletcher, and L. Rowe. 2006. The shaping of senescence in the wild. Trends in Ecology and Evolution 21:458-463.

Zajitschek, F., J. Hunt, S. R. K. Zajitschek, M. D. Jennions, and R. Brooks. 2007. No intra-locus sexual conflict over reproductive fitness or ageing in field crickets. PLoS ONE 2(1)e155. [doi: 10.1371/journal.pone.0000155]

Zuk, M., and G. R. Kolluru. 1998. Exploitation of sexual signals by predators and parasitoids. Quarterly Review of Biology 73:415-438.

\section{SUPPLEMENT}

Mathematica 6 notebook containing models used in the analysis (Ecological Archives E090-117-S1). 